\title{
Half self-convolution of the $k$-Fibonacci sequence
}

\author{
Sergio Falcon \\ Department of Mathematics, University of Las Palmas de Gran Canaria \\ Campus de Tafira, 35017 - Las Palmas de Gran Canaria, Spain \\ e-mail: sergio.falcon@ulpgc.es
}

Received: 21 March 2019

Revised: 21 July 2020

Accepted: 19 August 2020

\begin{abstract}
We say the $k$-Fibonacci numbers $F_{k, i}$ and $F_{k, j}$ are equidistant if $j=n-i$ and then we study some properties of these pairs of numbers. As a main result, we look for the formula to find the generating function of the product of the equidistant numbers, their sums and their binomial transforms. Next we apply this formula to some simple cases but more common than the general. In particular, we define the half self-convolution of the $k$-Fibonacci and $k$-Lucas sequences. Finally, we study the sum of these new sequences, their recurrence relations, and their generating functions.
\end{abstract}

Keywords: $k$-Fibonacci and $k$-Lucas numbers, Binet identity, Generating function, Convolution, Binomial transform.

2010 Mathematics Subject Classification: 11B37, 11B39, 11B65.

\section{Introduction}

For any integer number $k \geq 1$, the $k$-Fibonacci and the $k$-Lucas sequences are defined recurrently by $F_{k, n+1}=k F_{k, n}+F_{k, n-1}$ and $L_{k, n+1}=k L_{k, n}+L_{k, n-1}$, with initial conditions $F_{k, 0}=0$, $F_{k, 1}=1$ and $L_{k, 0}=2, L_{k, 1}=k$, respectively.

Generating function of the $k$-Fibonacci numbers is $f(k, x)=\frac{x}{1-k x-x^{2}}$ and for the $k$-Lucas numbers $l(k, x)=\frac{2-k x}{1-k x-x^{2}}$. Binet identity is $F_{k, n}=\frac{\sigma_{1}^{n}-\sigma_{2}^{n}}{\sigma_{1}-\sigma_{2}}$ being $\sigma_{1,2}=\frac{k \pm \sqrt{k^{2}+4}}{2}$ and $L_{k, n}=\sigma_{1}^{n}+\sigma_{2}^{n}$, respecitvely. Moreover $F_{k,-n}=(-1)^{n+1} F_{k, n}$ and $L_{k,-n}=(-1)^{n} L_{k, n}$. Finally, these $k$-numbers are related by the relations $L_{k, n}=F_{k, n-1}+F_{k, n+1}$ and $F_{k, n}=\frac{L_{k, n-1}+L_{k, n+1}}{\sqrt{k^{2}+4}}$. For more properties, see $[4,5]$ and $[1]$. 
In $[2,4]$ the following formulas are proven:

$$
\begin{aligned}
\sum_{i=0}^{m} L_{k, r i+p} & =\frac{L_{k, r(m+1)+p}-(-1)^{r} L_{k, r m+p}+(-1)^{p} L_{k, r-p}-L_{k, p}}{L_{k, r}-(-1)^{r}-1} \\
\sum_{i=0}^{m}(-1)^{i} L_{k, r i+p} & =\frac{(-1)^{m} L_{k, r(m+1)+p}+(-1)^{r+m} L_{k, r m+p}+(-1)^{m} L_{k, r-p}+L_{k, p}}{L_{k, r}+(-1)^{r}+1} \\
& \rightarrow \sum_{j=0}^{n} L_{k, j}=\frac{1}{k}\left(L_{k, n+1}+L_{k, n}+k-2\right) \\
F_{k, p} F_{k, q} & =\frac{1}{k^{2}+4}\left(L_{k, p+q}-(-1)^{q} L_{k, p-q}\right) \\
q=1 \rightarrow F_{k, p} & =\frac{1}{k^{2}+4}\left(L_{k, p+1}+L_{k, p-1}\right) \\
L_{k, p} L_{k, q} & =L_{k, p+q}+(-1)^{q} L_{k, p-q} \\
\sum_{j=0}^{n}(-1)^{j} L_{k, n-2 j} x^{j} & =\frac{L_{k, n}+L_{k, n+2} x}{1+\left(k^{2}+2\right) x+x^{2}} \text { (Generating function) }
\end{aligned}
$$

\section{On the equidistant $k$-Fibonacci and $k$-Lucas numbers}

Let $r \in N-\{0\}$ be. Given the finite sequence $\left\{a_{0}, a_{1}, \ldots a_{n}\right\}$, we say the elements $a_{r i}$ and $a_{n-r i}$ are equidistant.

Let us consider the finite sequence $E_{n}=\left\{F_{k, j} F_{k, n-j}\right\}$ for $j=1,2, \ldots, n / 2$.

Theorem 2.1. Sequence $E_{n}$ increases and decreases term to term until $F_{k,(n-1) / 2} F_{k,(n+1) / 2}$ or $F_{k, n / 2}^{2}$ according to $n$ is odd or even, respectively.

Proof. If in the formula (3) it is $p=i$ and $q=n-i, F_{k, i} F_{k, n-i}=\frac{1}{k^{2}+4}\left(L_{k, n}-(-1)^{n-i} L_{k, 2 i-n}\right)$, that is

$$
F_{k, i} F_{k, n-i}=\frac{1}{k^{2}+4}\left(L_{k, n}-(-1)^{i} L_{k, n-2 i}\right)
$$

because $L_{k .-r}=(-1)^{r} L_{k, r}$. So,

$$
\begin{aligned}
F_{k, 2 j} F_{k, n-2 j} & =\frac{1}{k^{2}+4}\left(L_{k, n}-L_{k, n-4 j}\right) \\
F_{k, 2 j+1} F_{k, n-2 j-1} & =\frac{1}{k^{2}+4}\left(L_{k, n}+L_{k, n-4 j-2}\right) \\
F_{k, 2 j-1} F_{k, n-2 j+1} & =\frac{1}{k^{2}+4}\left(L_{k, n}+L_{k, n-4 j+2}\right) \\
& \rightarrow F_{k, 2 j} F_{k, n-2 j}<F_{k, 2 j+1} F_{k, n-2 j-1} \\
L_{k, n-4 j+2}>L_{k, n-4 j-2} & \rightarrow F_{k, 2 j+1} F_{k, n-2 j-1}<F_{k, 2 j-1} F_{k, n-2 j+1} \\
& \rightarrow F_{k, 2 j} F_{k, n-2 j}<F_{k, 2 j+1} F_{k, n-2 j-1}<F_{k, 2 j-1} F_{k, n-2 j+1} .
\end{aligned}
$$


- If we apply the formula (6) to the equation (7), the generating function $e q(k, x)$ of the sequence of the products of the equidistant $k$-Fibonacci numbers $\left\{F_{k, i} F_{k, n-i}\right\}$ is

$$
\begin{aligned}
e q(k, x) & =\frac{1}{k^{2}+4}\left(L_{k, n}-p a(k, x)\right) \\
& =\frac{1}{k^{2}+4}\left(L_{k, n}-\frac{L_{k, n}+L_{k, n+2} x}{1+\left(k^{2}+2\right) x+x^{2}}\right) \\
& =\frac{1}{k^{2}+4} \frac{L_{k, n-2} x+L_{k, n} x^{2}}{1+\left(k^{2}+2\right) x+x^{2}} .
\end{aligned}
$$

Remark. To find the elements $\left\{F_{k, i} F_{k, n-i}\right\}_{0 \leq i \leq n}$ for $n=10$ and $k=1,2,3$ with Mathematica ${ }^{\circledR}$, first we must divide and then take into account $\frac{D}{d}=q+\frac{r}{d}$. Next, we can use the following little program:

$f\left[k_{-}, n_{-}\right]:=$Fibonacci $[n, k]$

$l\left[k_{-}, n_{-}\right]:=\operatorname{LucasL}[n, k]$

$n=10$

Table[Table $[f[k, i] * f[k, n-i],\{i, 0, n\}],\{k, 3\}]$

$p e\left[k_{-}, x_{-}\right]:=\frac{l[k, n]+l[k, n+2] x}{1+\left(k^{2}+2\right) x+x^{2}}$

Table $\left[\frac{1}{k^{2}+4}(l[k, n]-\right.$ CoefficientList[Series $\left.\left.[p e[k, x],\{x, 0, n\}], x]\right),\{k, 3\}\right]$

- From the formula (5), the product of the equidistant $k$-Lucas numbers is $L_{k, i} L_{k, n-i}=$ $L_{k, n}+(-1)^{i} L_{k, n-2 i}$. So, its generating function is

$$
\operatorname{eql}(k, x)=L_{k, n}+\frac{L_{k, n}+L_{k, n+2} x}{1+\left(k^{2}+2\right) x+x^{2}} .
$$

\section{Self-convolution of $k$-Fibonacci and $k$-Lucas sequences}

In this section we will study the sum of the equidistant $k$-Fibonacci and $k$-Lucas numbers. First we will proof the $k$-Lucas numbers verify the following lemmas.

Lemma 3.1. $L_{k, a n+2 r}-(-1)^{r} L_{k, a n}=\left(k^{2}+4\right) F_{k, r} F_{k, a n+r}$.

Proof. Taking into account the Binet identity and $\sigma_{1} \sigma_{2}=-1$,

$$
\begin{aligned}
& L_{k, a n+2 r}-(-1)^{r} L_{k, a n}=\sigma_{1}^{a n+2 r}+\sigma_{2}^{a n+2 r}-\left(\sigma_{1} \sigma_{2}\right)^{r}\left(\sigma_{1}^{a n}+\sigma_{2}^{a n}\right) \\
& =\sigma_{1}^{a n+r}\left(\sigma_{1}^{r}-\sigma_{2}^{r}\right)+\sigma_{2}^{a n+r}\left(\sigma_{2}^{r}-\sigma_{1}^{r}\right)=\left(\sigma_{1}^{r}-\sigma_{2}^{r}\right)\left(\sigma_{1}^{a n+r}-\sigma_{2}^{a n+r}\right) \\
& =\left(\sigma_{1}-\sigma_{2}\right)^{2} F_{k, r} F_{k, a n+r}=\left(k^{2}+4\right) F_{k, r} F_{k, a n+r} .
\end{aligned}
$$

Lemma 3.2. $L_{k, 2 r}-2(-1)^{r}=\left(k^{2}+4\right) F_{k, r}^{2}$.

Proof.

$$
\begin{aligned}
L_{k, 2 r}-2(-1)^{r} & =\sigma_{1}^{2 r}+\sigma_{2}^{2 r}-2\left(\sigma_{1} \sigma_{2}\right)^{r}=\sigma_{1}^{r}\left(\sigma_{1}^{r}-\sigma_{2}^{r}\right)+\sigma_{2}^{r}\left(\sigma_{2}^{r}-\sigma_{1}^{r}\right) \\
& =\left(\sigma_{1}^{r}-\sigma_{2}^{r}\right)^{2}=\left(k^{2}+4\right) F_{k, r}^{2}
\end{aligned}
$$


Then, we can prove next theorem.

Theorem 3.3 (Sum of the products $L_{k, r i} L_{k, n-r i}$ ). Let us consider the finite sequence $\left\{L_{k, 0}, L_{k, 1}, L_{k, 2}, \ldots L_{k, n-1}, L_{k, n}\right\}$. The sum of the equidistant $k$-Fibonacci numbers is

$$
\sum_{j=0}^{n} L_{k, r j} L_{k, n-r j}=(n+1) L_{k, n}+\frac{(-1)^{n(r+1)} F_{k,(2 r-1) n+r}+F_{k, n+r}}{F_{k, r}} .
$$

Proof. If $p=n-r$ and $q=r$ in the formula (5), then

$L_{k, r} L_{k, n-r}=L_{k, n}+(-1)^{r} L_{k, n-2 r}$. So,

$$
\sum_{j=0}^{n} L_{k, r j} L_{k, n-r j}=\sum_{j=0}^{n}\left(L_{k, n}+(-1)^{r j} L_{k, n-2 r j}\right)=(n+1) L_{k, n}+\sum_{j=0}^{n}(-1)^{r j} L_{k, n-2 r j} .
$$

Next we will apply the formulas (1) and (2) with $m=-2 r$ and $p=n$, and $L_{k,-a}=(-1)^{a} L_{k, a}$. If $r$ is even:

$$
\begin{aligned}
\sum_{j=0}^{n}(-1)^{r j} L_{k, n-2 r j} & =\sum_{j=0}^{n} L_{k, n-2 r j} \\
& =\frac{L_{k, n-2 r(n+1)}-L_{k, n-2 r n}+L_{k, n+2 r}-L_{k, n}}{L_{k, 2 r}-2} \\
& =\frac{(-1)^{n} L_{k,(2 r-1) n+2 r}-(-1)^{n} L_{k,(2 r-1) n}+L_{k, n+2 r}-L_{k, n}}{L_{k, 2 r}-2}
\end{aligned}
$$

If $r$ is odd:

$$
\begin{aligned}
\sum_{j=0}^{n}(-1)^{r j} L_{k, n-2 r j} & =\sum_{j=0}^{n}(-1)^{j} L_{k, n-2 r j} \\
& =\frac{(-1)^{n} L_{k, n-2 r(n+1)}+(-1)^{n} L_{k, n-2 r n}+L_{k, n+2 r}+L_{k, n}}{L_{k, 2 r}+2} \\
& =\frac{L_{k,(2 r-1) n+2 r}+L_{k,(2 r-1) n}+L_{k, n+2 r}+L_{k, n}}{L_{k, 2 r}+2}
\end{aligned}
$$

We can join these last two formulas and $L_{k,-r}=(-1)^{r} L_{k, r}$, to obtain

$\sum_{j=0}^{n} L_{k, r j} L_{k, n-r j}=$

$(n+1) L_{k, n}+\frac{(-1)^{n(r+1)} L_{k,(2 r-1) n+2 r}-(-1)^{n(r+1)+r} L_{k,(2 r-1) n}+L_{k, n+2 r}-(-1)^{r} L_{k, n}}{L_{k, 2 r}-2(-1)^{r}}$.

Now, if we will apply Lemma 1 and Lemma 2,

$$
\begin{aligned}
L_{k,(2 r-1) n+2 r}-(-1)^{r} L_{k,(2 r-1) n} & =\left(k^{2}+4\right) F_{k, r} F_{k,(2 r-1) n+r} \\
L_{k, n+2 r}-(-1)^{r} L_{k, 2 n} & =\left(k^{2}+4\right) F_{k, r} F_{k, n+r} \\
L_{k, 2 r}-2(-1)^{r} & =\left(k^{2}+4\right) F_{k, r}^{2}
\end{aligned}
$$

So,

$$
\sum_{j=0}^{n} L_{k, r j} L_{k, n-r j}=(n+1) L_{k, n}+\frac{(-1)^{n(r+1)} F_{k,(2 r-1) n+r}+F_{k, n+r}}{F_{k, r}} .
$$


In particular, if $r=1, T_{n}=\sum_{j=0}^{n} L_{k, j} L_{k, n-j}=(n+1) L_{k, n}+2 F_{k, n+1}$.

A sequence of sums $\sum_{j=0}^{n} L_{k, j} L_{k, n-j}$ is called the self-convolution of the sequence $\left\{L_{k, n}\right\}_{n \in N}$. For $k=1,2,3$, the first few terms of these sequences are:

- $\{4,4,13,22,45,82,152,274,491,870 \ldots\}$ is the sequence A099924 in OEIS [6];

- $\{4,8,28,80,228,632,1724,4640,12356,32616 \ldots\}$;

- $\{4,12,53,210,813,3078,11464,42150,153371 \ldots\}$.

\subsection{Generating function and recurrence relation for the self-convolution of the $k$-Lucas sequence}

- Taking into account $l(k, x)=\frac{2-k x}{1-k x-x^{2}}$ is the generating function of the $k$-Lucas numbers, $l(k, x)^{2}$ is the generating function of the self-convolution of $\left\{L_{k, n}\right\}$, [7, Rule 3].

- The denominator of the generating function of the self-convolution $L_{k} \otimes L_{k}$ is $\left(1-k x-x^{2}\right)^{2}=1-2 k x+\left(k^{2}-2\right) x^{2}+2 k x^{3}+x^{4}$ so the recurrence relation for this sequence is $T_{n}=2 k T_{n-1}-\left(k^{2}-2\right) T_{n-2}-2 k T_{n-3}-T_{n-4}$ for $n \geq 4$, with initial conditions $T_{0}=4, T_{1}=4 k, T_{2}=5 k^{2}+8$, and $T_{3}=6 k^{3}+16 k$.

\subsection{Half self-convolution of the $k$-Lucas sequence}

In the sequence $\left\{L_{k, j} L_{k, n-j}\right\}$, only the first $\left\lfloor\frac{n+1}{2}\right\rfloor$ terms are different. The sum of these numbers is called the half self-convolution of the $k$-Lucas numbers: $T_{n / 2}=\sum_{j=0}^{n / 2} L_{k, j} L_{k, n-j}$.

Then $T_{n / 2}=\frac{T_{n}}{2}$ if $n$ is odd, $n=2 p+1$, and $T_{n / 2}=\frac{T_{n}+L_{k, p}^{2}}{2}$ if $n=2 p$, because there are $2 p+2$ products $\left\{L_{k, i} L_{k, n-i}\right\}$ but only $p+1$ different products of equidistant $k$-Lucas numbers if $n$ is odd and $2 p$ products equal two to two plus only one $L_{k, p}^{2}$ if $n$ is even. So, if $\eta(n)=\frac{(-1)^{n}+1}{2}$.

$$
\sum_{j=1}^{n / 2} L_{k, j} L_{k, n-j}=\frac{1}{2}\left((n+1) L_{k, n}+2 F_{k, n+1}+\eta(n) L_{k, n / 2}^{2}\right)
$$

Theorem 3.4. Sum of the products $F_{k, r i} F_{k, n-r i}$ in the finite sequence $\left\{F_{k, 0}, F_{k, 1}, F_{k, 2}, \ldots, F_{k, n-2}, F_{k, n-1}, F_{k, n}\right\}$ is

$$
\sum_{j=0}^{n} F_{k, r j} F_{k, n-r j}=\frac{1}{k^{2}+4}\left((n+1) L_{k, n}-\frac{(-1)^{n(r+1)} F_{k,(2 r-1) n+r}+F_{k, n+r}}{F_{k, r}}\right) .
$$

Taking into account $F_{k, r j} F_{k, n-r j}=\frac{L_{k, n}-(-1)^{n-r j} L_{k, 2 r j-n}}{k^{2}+4}$, we can prove this theorem in a similar form to the used for the $k$-Lucas numbers. 
Moreover, if $r=1$, as $L_{k, n}=F_{k, n+1}+F_{k, n-1}$,

$$
E_{k, n}=\frac{1}{k^{2}+4}\left((n+1) F_{k, n-1}+(n-1) F_{k, n+1}\right)
$$

The sequence of sums $\sum_{j=0}^{n} F_{k, j} F_{k, n-j}$ is called self-convolution of the $k$-Fibonacci sequence.

Next we show the first terms of these sequences for $k=1,2,3$ :

- $\{0,1,2,5,10,20,38,71,130,235 \ldots\}, \mathrm{A} 001629$;

- $\{0,1,4,14,44,131,376,1052,2888 \ldots\}, A 006645$;

- $\{0,1,6,29,126,516,2034,7807,29382 \ldots\}$.

3.3 Recurrence relation on the sequence $\left\{E_{k, n}\right\}=\left\{\sum_{j=0}^{n} F_{k, j} F_{k, n-j}\right\}$

From equation (10)

$$
\begin{aligned}
\left(k^{2}+4\right) E_{k, n+1} & =\left(k^{2} n+2 n+2\right) F_{k, n}+k n F_{k, n-1} \\
\left(k^{2}+4\right) E_{k, n} & =k(n-1) F_{k, n}+2 n F_{k, n-1} \\
\left(k^{2}+4\right) E_{k, n-1} & =2(n-1) F_{k, n}-k n F_{k, n-1}
\end{aligned}
$$

This linear system has a single unique solution if and only if the augmented determinant is null:

$$
\begin{gathered}
\left|\begin{array}{ccc}
k^{2} n+2 n+2 & k n & \left(k^{2}+4\right) E_{k, n+1} \\
k(n-1) & 2 n & \left(k^{2}+4\right) E_{k, n} \\
2(n-1) & -k n & \left(k^{2}+4\right) E_{k, n-1}
\end{array}\right|=0 \\
\rightarrow(n+1) E_{k, n+1}+k n E_{k, n}-(n-1) E_{k, n-1}=0 .
\end{gathered}
$$

So, the terms of the sequence $\left\{E_{k, n}\right\}=\left\{\sum_{j=0}^{n} F_{k, j} F_{k, n-j}\right\}$ verify the recurrence relation $E_{k, n+1}=\frac{1}{n-1}\left(k n E_{k, n}+(n+1) E_{k, n-1}\right)$ for $n \geq 2$ with initial conditions $E_{k, 1}=0$ and $E_{k, 2}=1$.

\subsection{Sum of the equidistant $k$-Fibonacci numbers}

By the same reason that in Theorem 3.2, in the sequence $\left\{F_{k, j} F_{k, n-j}\right\}$, only the first $\left\lfloor\frac{n+1}{2}\right\rfloor$ terms are different. The sum of these numbers is called the half self-convolution of the $k$-Fibonacci numbers so the sum of the products of the equidistant $k$-Fibonacci numbers $\sum_{j=0}^{n / 2} F_{k, j} F_{k, n-j}$ in the finite sequence $\left\{F_{k, j}\right\}_{0 \leq j \leq n}$ is $T_{k, n}=\frac{E_{k, n}}{2}$ for $n$ odd and $T_{k, n}=\frac{E_{k, n}+F_{k, n / 2}^{2}}{2}$ for $n$ even. Then, both equations can be join in the next:

$$
\sum_{j=0}^{n / 2} F_{k, j} F_{k, n-j}=\frac{1}{2}\left(\frac{(n+1) F_{k, n-1}+(n-1) F_{k, n+1}}{k^{2}+4}+\eta(n) F_{k, n / 2}^{2}\right)
$$


Consequently, $T_{k}$ for $n=1,2,3 \ldots$ is the half-convolution of the $k$-Fibonacci sequence $F_{k}-\{0\}$ with itself or the half self-convolution of the $k$-Fibonacci sequence. First elements of these sequences, for $k=1,2,3$ are:

- $T_{1}=\{0,1,1,3,5,12,19,40, \ldots\}: \mathrm{A} 024458$;

- $T_{2}=\{0,1,2,9,22,78,188,598, \ldots\}$;

- $T_{3}=\{0,1,3,19,63,308,1017,4448, \ldots\}$.

\subsection{Generating function of the half self-convolution of the sequence $\left\{F_{k, n}\right\}$}

In this section, we must take into account the formula (11).

- Generating function of the $k$-Fibonacci sequence $F_{k}$ is $f_{k}(x)=\frac{x}{1-k x-x^{2}}$ [5]. So, $u_{k}(x)=\frac{1}{1-k x-x^{2}}$ is the generating function of the sequence $F_{k}-\{0\}$ and then $\left(u_{k}(x)\right)^{2}$ is the generating function of the convolution of the sequence $F_{k}-\{0\}$ with itself:

1. $U_{1}=\{1,2,5,10,20,38 \ldots\}:$ A001629 in OEIS.

2. $U_{2}=\{1,4,14,44,1331,376 \ldots\}:$ A006645 in OEIS.

3. $U_{3}=\{1,6,29,126,516,2034 \ldots\}$

- Generating function of the sequence of squares of the $k$-Fibonacci numbers is $v_{k}(x)=\frac{x(1-x)}{1-\left(k^{2}+1\right)\left(x+x^{2}\right)+x^{3}}$ [3]. Then, the function $v_{k}\left(x^{2}\right)$ is the generating function of the sequence whose even terms are the squares of the $k$-Fibonacci numbers and the odd terms are null: $\left\{0,1,0, k^{2}, 0,\left(k^{2}+1\right)^{2}, 0,\left(k^{3}+2 k\right)^{2}, 0, \ldots\right\}$

- Consequently, and according to the formula (11), $t_{k}(x)=\frac{1}{2}\left(\left(u_{k}(x)\right)^{2}+v_{k}\left(x^{2}\right)\right)$ is the generating function of the sequences of sums of the products of the equidistant $k$-Fibonacci numbers $T_{k}=\left\{T_{k, n}\right\}$.

\subsection{Generating function of the half self-convolution of the sequence $\left\{L_{k, n}\right\}$}

We will follow the same process as in the previous paragraph.

- $U_{k}(x)=\frac{2-k x}{1-k x-x^{2}}$ is the generating function of the $k$-Lucas sequence $L_{k}=\left\{L_{k, n}\right\}$. So, $\left(U_{k}(x)\right)^{2}$ is the generating function of the self-convolution of $L_{k}$. Only the self-convolution $L_{1} \otimes L_{1}=\{4,4,13,22,45,82,152,274, \ldots\}$ is in OEIS (A099924).

- The $k$-Lucas numbers verify the relation $L_{k, n}^{2}=\left(k^{2}+1\right)\left(L_{k, n-1}^{2}+L_{k, n-2}^{2}\right)-L_{k, n-3}^{2}$ (the same as the $k$-Fibonacci numbers).

Then, the denominator of its generating function is $1-\left(k^{2}+1\right)\left(x+x^{2}\right)+x^{3}$, being the numerator $L_{k, 0}^{2}+\left(L_{k, 1}^{2}-\left(k^{2}+1\right) L_{k, 0}^{2}\right) x+\left(L_{k, 2}^{2}-\left(k^{2}+1\right)\left(L_{k, 1}^{2}+L_{k, 0}^{2}\right)\right) x^{2}$. 
So, $V_{k}(x)=\frac{4-\left(3 k^{2}+4\right)-k^{2} x^{2}}{1-\left(k^{2}+1\right)\left(x+x^{2}\right)+x^{3}}$ is the generating function of the squares of the $k$-Lucas numbers and $\left(V_{k}(x)\right)^{2}$ is the generating function of sequence whose even terms are the squares of the $k$-Lucas numbers and the odd terms are null.

- Then $W_{k}(x)=\frac{1}{2}\left(\left(U_{k}(x)\right)^{2}+V_{k}\left(x^{2}\right)\right)$ is the generating function of the half self-convolution of the $k$-Lucas sequence.

\section{Binomial transform of the products of equidistant $k$-Fibonacci and $k$-Lucas numbers}

Next we will study the binomial transform of the products of equidistant $k$-Fibonacci and $k$-Lucas numbers.

Theorem 4.1. Binomial transform of the finite sequence of products $L_{k, r j} L_{k, n-r j}$ is

$$
\sum_{j=0}^{n}\left(\begin{array}{l}
n \\
j
\end{array}\right) L_{k, r j} L_{k, n-r j}=2^{n} L_{k, n}+L_{k, r}^{n} L_{k, n-r n}
$$

Proof.

$$
\begin{aligned}
& \sum_{j=0}^{n}\left(\begin{array}{l}
n \\
j
\end{array}\right) L_{k, r j} L_{k, n-r j}=\sum_{j=0}^{n}\left(\begin{array}{c}
n \\
j
\end{array}\right)\left(\sigma_{1}^{r j}+\sigma_{2}^{r j}\right)\left(\sigma_{1}^{n-r j}+\sigma_{2}^{n-r j}\right) \\
& =\sum_{j=0}^{n}\left(\begin{array}{c}
n \\
j
\end{array}\right)\left(\sigma_{1}^{n}+\sigma_{2}^{n}+\sigma_{1}^{n}\left(\frac{\sigma_{2}}{\sigma_{1}}\right)^{r j}+\sigma_{2}^{n}\left(\frac{\sigma_{1}}{\sigma_{2}}\right)^{r j}\right) \\
& =\sum_{j=0}^{n}\left(\begin{array}{c}
n \\
j
\end{array}\right) L_{k, n}+\sigma_{1}^{n} \sum_{j=0}^{n}\left(\begin{array}{c}
n \\
j
\end{array}\right)\left(\frac{\sigma_{2}}{\sigma_{1}}\right)^{r j}+\sigma_{2}^{n} \sum_{j=0}^{n}\left(\begin{array}{c}
n \\
j
\end{array}\right)\left(\frac{\sigma_{1}}{\sigma_{2}}\right)^{r j} \\
& =L_{k, n} \sum_{j=0}^{n}\left(\begin{array}{c}
n \\
j
\end{array}\right)+\sigma_{1}^{n}\left(1+\left(\frac{\sigma_{2}}{\sigma_{1}}\right)^{r}\right)^{n}+\sigma_{2}^{n}\left(1+\left(\frac{\sigma_{1}}{\sigma_{2}}\right)^{r}\right)^{n} \\
& =2^{n} L_{k, n}+\sigma_{1}^{n}\left(\frac{\sigma_{1}^{r}+\sigma_{2}^{r}}{\sigma_{1}^{r}}\right)^{n}+\sigma_{2}^{n}\left(\frac{\sigma_{1}^{r}+\sigma_{2}^{r}}{\sigma_{2}^{r}}\right)^{n} \\
& =2^{n} L_{k, n}+\left(\sigma_{1}^{r}+\sigma_{2}^{r}\right)^{n}\left(\sigma_{1}^{n-r n}+\sigma_{2}^{n-r n}\right) \\
& =2^{n} L_{k, n}+L_{k, r}^{n} L_{k, n-r n} .
\end{aligned}
$$

In particular,

- if $r=1$, it is $C_{p}=\sum_{j=0}^{n}\left(\begin{array}{l}n \\ j\end{array}\right) L_{k, j} L_{k, n-j}=2^{n} L_{k, n}+2 k^{n}$;

- if $r=2$, it is $\sum_{j=0}^{n}\left(\begin{array}{l}n \\ j\end{array}\right) L_{k, 2 j} L_{k, n-2 j}=\left(2^{n}+(-1)^{n}\left(k^{2}+2\right)^{n}\right) L_{k, n}$. 
We must take into account the formula (9), so the binomial transform of the products of equidistant $k$-Lucas numbers in the sequence $\left\{L_{k, n}\right\}$ is $C_{e}=2^{n-1} L_{k, n}+k^{n}+\frac{\eta}{2}\left(\begin{array}{c}n \\ n / 2\end{array}\right) L_{k, n / 2}^{2}$ with $\eta=\frac{(-1)^{n}+1}{2}$.

Taking into account this theorem and the Binet Identity, it is easy to prove the following theorem for the $k$-Fibonacci numbers.

Theorem 4.2. The binomial transform of the products $\left\{F_{k, r j} F_{k, n-r j}\right\}$ is

$$
\sum_{j=0}^{n}\left(\begin{array}{l}
n \\
j
\end{array}\right) F_{k, r j} F_{k, n-r j}=\frac{2^{n} L_{k, n}-L_{k, r}^{n} L_{k, n-r n}}{k^{2}+4} .
$$

In particular, if $r=1$, binomial transform of these products is

$$
D_{n}=\sum_{j=0}^{n}\left(\begin{array}{l}
n \\
j
\end{array}\right) F_{k, j} F_{k, n-j}=\frac{2^{n} L_{k, n}-2 k^{n}}{k^{2}+4}
$$

For $k=1,2,3$, the sequences obtained are:

- $\{0,2,6,22,70,230,742,2406,7782,25190,81510, \ldots\}$ : A014334 in OEIS;

- $\{0,2,12,64,320,1568,7616,36864,178176,860672,4156416, \ldots\}$;

- $\{0,2,18,134,930,6278,41874,277814,1838754,12156902,80335794, \ldots\}$.

Finally, binomial transform of the products of the equidistant $k$-Fibonacci numbers is

$$
V(n)=\sum_{j=0}^{n / 2}\left(\begin{array}{l}
n \\
j
\end{array}\right) F_{k, j} F_{k, n-j}=\frac{2^{n-1} L_{k, n}-k^{n}}{k^{2}+4}+\frac{1}{2} \eta(n)\left(\begin{array}{c}
n \\
n / 2
\end{array}\right) F_{k, n / 2}^{2}
$$

This binomial transform is also known as exponential half-convolution of a $k$-Fibonacci sequence with itself.

For $k=1,2,3$, the first few terms of the correspondent sequences are:

- $k=1 \rightarrow\{0,2,3,14,35,155,371, \ldots\}:$ A203578 in OEIS;

- $k=2 \rightarrow\{0,2,6,44,160,1034,3808, \ldots\}$;

- $k=3 \rightarrow\{0,2,9,94,465,4139,20397, \ldots\}$.

\subsection{Generating function of exponential self-convolution of the sequence $\left\{F_{k, n}\right\}$}

Remember that the generating function of the $k$-Lucas numbers is $l(x)=\frac{2-k x}{1-k x-x^{2}}$ and that of $\left\{k^{n}\right\}$ is $\frac{1}{1-k x}$. Formula (12) is $\sum_{k=1}^{n}\left(\begin{array}{l}n \\ j\end{array}\right) F_{k, j} F_{k, n-j}=\frac{2^{n} L_{k, n}-2 k^{n}}{k^{2}+4}$. Then, the generating function of $\sum_{k=1}^{n}\left(\begin{array}{l}n \\ j\end{array}\right) F_{k, j} F_{k, n-j}$ is 


$$
b f(x)=\frac{1}{k^{2}+4}\left(l(k x)-\frac{2}{1-k x}\right)=\frac{2 x^{2}}{1-3 k x+2\left(k^{2}-2\right) x^{2}+4 k x^{3}}
$$

Consequently, the generating function of the binomial transform of the equidistant $k$-Fibonacci numbers is

$$
s s(x)=\frac{x^{2}}{1-3 k x+2\left(k^{2}-2\right) x^{2}+4 k x^{3}}+\frac{1}{2} \eta(n)\left(\begin{array}{c}
n \\
n / 2
\end{array}\right) F_{k, n / 2}^{2}
$$

with $\eta(n)=\frac{(-1)^{n}+1}{2}$.

\subsection{Recurrence relation of exponential self-convolution of the sequence $\left\{F_{k, n}\right\}$}

Let

$$
\left\{E_{k, n}\right\}=\left\{\sum_{j=0}^{n}\left(\begin{array}{l}
n \\
j
\end{array}\right) F_{k, j} F_{k, n-j}\right\} .
$$

The denominator of the formula (13) shows that the recurrence relation for this sequence is

$$
E_{k, n}=3 k E_{k, n-1}-2\left(k^{2}-2\right) E_{k, n-2}-4 k E_{k, n-3}
$$

with initial conditions $E_{k, 1}=0, E_{k, 2}=2 ; E_{k, 3}=6 k$.

4.3 On the sequences $M_{k, n}=\sum_{j=0}^{n}\left(\begin{array}{l}n \\ j\end{array}\right) L_{k, n} L_{k, n-j}$

For $k=1,2,3$, the sequences $M_{k}$ are:

- $\{4,4,14,34,114,354, \ldots\}: 2 * A 203579$ in OEIS;

- $\{4,8,32,128,576,2688, \ldots\}: 4 * A 084137$ in OEIS;

- $\{4,12,62,342,2066,13062, \ldots\}$.

Taking into account the $k$-Fibonacci and the $k$-Lucas numbers have the same recurrence relation and only differ in the initial conditions, the equations for ones and others must have a similar form.

1. Recurrence relation. From the formula (14), the recurrence relation for these numbers is $M_{k, n}=3 k M_{k, n-1}-2\left(k^{2}-2\right) M_{k, n-2}-4 k M_{k, n-3}$ with initial conditions $M_{k, 0}=2$, $M_{k, 1}=4 k, M_{k, 2}=6 k^{2}+8$.

2. Generating function. Let $m(k, x)$ be the generating function of these sequences. The preceding formula shows the way forward to find the generating function. 


$$
\begin{array}{rlrl}
m(k, x) & =M_{k, 0}+M_{k, 1} x+M_{k, 2} x^{2}+M_{k, 3} x^{3}+\cdots \\
3 k x m(k, x) & = & 3 k M_{k, 0} x+3 k M_{k, 1} x^{2}+3 k M_{k, 2} x^{3}+\cdots \\
2\left(k^{2}-2\right) x^{2} m(k, x) & = & 2\left(k^{2}-2\right) M_{k, 0} x^{2}+2\left(k^{2}-2\right) M_{k, 1} x^{3}+\cdots \\
4 k x^{3} m(k, x) & = & 4 k M_{k, 0} x^{3}+\cdots
\end{array}
$$

$$
\begin{aligned}
& \left(1-3 k x+2\left(k^{2}-2\right) x^{2}+4 k x^{3}\right) m(k, x)= \\
& =M_{k, 0}+\left(M_{k, 1}-2 k M_{k, 0}\right) x+\left(M_{k, 2}-3 k M_{k, 1}+\left(2 k^{2}-4\right) M_{k, 0}\right) x^{2} \\
& \rightarrow m(k, x)=\frac{4-8 k x+\left(2 k^{2}-8\right) x^{2}}{1-3 k x+2\left(k^{2}-2\right) x^{2}+4 k x^{3}}
\end{aligned}
$$

\section{Conclusions}

We have found formulas to the sum of the products of $k$-Fibonacci and $k$-Lucas numbers as well its binomial transform. Next, we shown the sums of equidistant $k$-Fibonacci and $k$-Lucas numbers. And finally, we give the formulas of their generating functions and binomial transforms.

\section{References}

[1] Falcon, S. (2011) On the $k$-Lucas numbers, Int. J. Contemp. Math. Sciences, 6 (21), 1039-1050.

[2] Falcon, S. (2012) On the k-Lucas numbers of arithmetic indexes, Applied Mathematics, 3, 1202-1206.

[3] Falcon, S. (2014). On the Generating Functions of the Powers of the $k$-Fibonacci Numbers, Scholars Journal of Engineering and Technology (SJET), 2 (4C), 669-675.

[4] Falcon, S., \& Plaza, A. (2007). On the Fibonacci k-numbers, Chaos, Solit. \& Fract., 32 (5), 1615-1624.

[5] Falcon, S., \& Plaza, A. (2007). The $k$-Fibonacci sequence and the Pascal 2-triangle, Chaos, Solit. \& Fract., 33(1), 38-49.

[6] Sloane, N. J. A., editor. The On-Line Encyclopedia of Integer Sequences, Available online at: https://oeis.org

[7] Wilf, H. S. (1994). Generating functionology, Ed. Academic Press Inc., Available online at: http: //www. math.upenn.edu/ wilf/DownldGF. html. 